\title{
C. elegans PAT-9 is a nuclear zinc finger protein critical for the assembly of muscle attachments
}

\author{
Qian Liu', Takako I Jones ${ }^{1,2}$, Rebecca A Bachmann', Mitchell Meghpara', Lauren Rogowski ${ }^{1}$, \\ Benjamin D Williams ${ }^{1}$ and Peter $L$ Jones ${ }^{1,2^{*}}$
}

\begin{abstract}
Background: Caenorhabditis elegans sarcomeres have been studied extensively utilizing both forward and reverse genetic techniques to provide insight into muscle development and the mechanisms behind muscle contraction. A previous genetic screen investigating early muscle development produced 13 independent mutant genes exhibiting a Pat (paralyzed and arrested elongation at the two-fold length of embryonic development) muscle phenotype. This study reports the identification and characterization of one of those genes, pat- 9 .

Results: Positional cloning, reverse genetics, and plasmid rescue experiments were used to identify the predicted $C$. elegans gene T27B1.2 (recently named ztf-19) as the pat-9 gene. Analysis of pat-9 showed it is expressed early in development and within body wall muscle lineages, consistent with a role in muscle development and producing a Pat phenotype. However, unlike most of the other known Pat gene family members, which encode structural components of muscle attachment sites, PAT-9 is an exclusively nuclear protein. Analysis of the predicted PAT-9 amino acid sequence identified one putative nuclear localization domain and three $\mathrm{C} 2 \mathrm{H} 2$ zinc finger domains. Both immunocytochemistry and PAT-9::GFP fusion expression confirm that PAT-9 is primarily a nuclear protein and chromatin immunoprecipitation (ChIP) experiments showed that PAT-9 is present on certain gene promoters.

Conclusions: We have shown that the T27B1.2 gene is pat-9. Considering the Pat-9 mutant phenotype shows severely disrupted muscle attachment sites despite PAT-9 being a nuclear zinc finger protein and not a structural component of muscle attachment sites, we propose that PAT-9 likely functions in the regulation of gene expression for some necessary structural or regulatory component(s) of the muscle attachment sites.
\end{abstract}

Keywords: Sarcomere, Muscle, Zinc finger, Pat

\section{Introduction}

The nematode C. elegans provides an established, developmentally well-documented, and evolutionarily conserved system to study muscle structure, development, and function $[1,2]$. The C. elegans sarcomere, the basic muscle contraction unit, has been studied for decades revealing a highly organized structure consisting of several hundred proteins, yet new components are still being identified [26]. In C. elegans sarcomeres, myosin thick filaments are organized around M-lines and actin thin filaments are

\footnotetext{
*Correspondence: pjones@bbri.org

'Department of Cell and Developmental Biology, University of Illinois at Urbana-Champaign, 601 S. Goodwin Ave, B107 Chemical and Life Sciences Laboratory, Urbana, IL 61801, USA

${ }^{2}$ Present Address: Boston Biomedical Research Institute, 64 Grove St. Watertown, MA 02472, USA
}

anchored to the dense bodies, structures analogous to the vertebrate $\mathrm{Z}$-disk. The dense bodies and $\mathrm{M}$-lines are sites of attachment for body wall muscle cells to the basement membrane, thus transmitting the force of muscle contraction and allowing movement [7]. The overall mechanism of C. elegans muscle function is highly evolutionarily conserved and many of the known proteins have vertebrate orthologs within vertebrate muscle costameres or nonmuscle focal adhesions $[1,2,6,8]$.

Many of the components necessary for C. elegans muscle attachments were identified by immunological approaches or through genetic screening for mutants exhibiting disorganized myofilaments, paralysis, and/or embryonic arrest $[4,9,10]$. Genes required for muscle development and function are grouped into two main phenotypic classes of mutants, Pat (paralyzed and

\section{Biomed Central}


arrested elongation at the two-fold length of embryonic development) and Unc (Uncoordinated), with some genes capable of producing both phenotypes depending on the nature of the mutation $[10,11]$. Both of these phenotypic classes comprise proteins that localize specifically to the M-lines, dense bodies or both, and much of their organized assembly into functional sarcomeres has been characterized (reviewed in [1,7]). A fourth type of localization for some sarcomeric proteins is exemplified by ZYX-1, UNC-95, UNC-97, and UNC-98, which are found both at the sarcomere and in the nucleus, supporting an additional role for the sarcomere as a platform for mediating signal transduction to the nucleus to influence gene expression [12-15].

In a continuing effort to identify new components required for muscle attachment site assembly, we have focused on characterizing the Pat group of C. elegans mutants. Mutations in the genes that encode the membrane-associated components of the muscle attachment sites, such as unc-52, pat-2, pat-3, unc-112, unc-97, pat4 and pat-6, display the most severe Pat phenotype, in which neither thin filaments nor thick filaments are assembled into the myofilament lattice [10]. Previous studies indicate that PAT-4, UNC-97 and PAT-6 serve as intermediary members of the linkage formed between the integrins and myofilaments [16-18]. Here, we identified and characterized a novel gene in the Pat family, pat-9 $[10,19]$. In pat-9 mutants, the recruitment of both actin thin filaments and myosin thick filaments to the muscle cell membrane appears to be disrupted. Similar to other Pat genes, pat-9 is expressed in C. elegans body wall muscle during embryogenesis. Unlike other genes in the Pat family that are structural and functional components of muscle attachments, pat-9 encodes a nuclear localized $\mathrm{C} 2 \mathrm{H} 2$ zinc finger transcription factor, suggesting a potential regulatory role, as opposed to a sarcomeric structural function for PAT-9.

\section{Results}

\section{The pat-9 gene is encoded by T27B1.2 (ztf-19)}

The pat- 9 gene was genetically mapped to the right end of the X chromosome [19]. Subsequently, a combination of positional cloning and candidate gene approaches were used to identify the pat-9 gene. Single nucleotide polymorphism (SNP) mapping data localized pat-9(st558) to the right of the F38E9 marker on chromosome X, near the T25D1 marker, since no recombination events were detected between this marker and pat-9 (Additional file 1: Table S1). There are 23 genes in the $200 \mathrm{~kb}$ region centered at T25D1, of which 9 are expressed in muscle based on SAGE tag data (Figure 1A and Additional file 1: Table S2) from the WormBase website (http://www.wormbase. org, Release WS223). Therefore, RNA interference

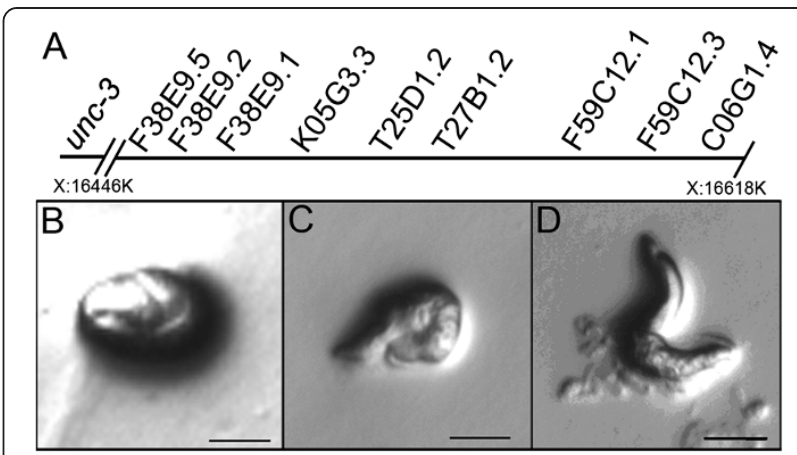

Figure 1 RNAi for T27B1.2 produces a Pat phenotype. A) Nine candidate genes located near the mapped pat-9 locus on the $X$ chromosome show muscle expression patterns according to SAGE data. B) Wild type N2 animals at the 2.5-fold stage C) pat-9 mutant animals segregated from strain RW1385. D) Only T27b1.2 RNAi caused a Pat phenotype at the embryonic stage. Bars $=10 \mu \mathrm{m}$.

(RNAi) was performed on each of the nine candidate genes. The RNAi for T27B1.2 was the only one that produced a significant number $(10 \%, \mathrm{~N}>200)$ of phenotypic Pat-like F1 progenies (Figure 1D compared with 1B, N2 and 1 C pat-9). Performing RNAi for the other eight candidate genes resulted in normal embryos. Our data was recently corroborated by Meissner et al. who conducted an RNAi screen for new Pat genes and identified T27B1.2 as having a Pat loss of function phenotype [20]. This data strongly implicates T27B1.2 as pat-9.

The hypothetical gene T27B1.2, identified by the $C$. elegans genome-sequencing consortium near the right end of chromosome $\mathrm{X}$, is predicted to encode a $\mathrm{C} 2 \mathrm{H} 2$ zinc finger protein and was recently placed into the zinc finger transcription factor family and renamed $z t f$ 19; however, the gene will be referred to as T27B1.2. To confirm T27B1.2 is pat-9, a transformation rescue experiment was performed. Injection of a genomic DNA fragment containing T27B1.2 and $3.3 \mathrm{~kb}$ of upstream noncoding genomic DNA completely rescued the phenotype of pat-9 homozygotes indicating the causal mutation resides in the T27B1.2 gene. To identify the pat-9(st558) mutation at the molecular level, the T27B1.2 gene was amplified and sequenced from pat-9(st558), segregated by strain RW1385. DNA sequence analysis revealed a single point mutation $(\mathrm{G} \rightarrow \mathrm{A})$ in the putative splice donor site of the $3 \mathrm{rd}$ intron (Figure $2 \mathrm{~A}$ and $\mathrm{B}$ ). The $\mathrm{G}$ residue at this position is completely conserved $(\sim 100 \%)$ in C. elegans splice donor sites, and mutation of this $G$ residue is predicted to lead to aberrant splicing resulting in a nonfunctional PAT-9 protein. However, since the splice junctions are predicted based on a gene model and had not yet been verified, total RNA was extracted from both pat-9 mutant animals and N2 animals and 
subjected to reverse transcriptase PCR to amplify T27B1.2 cDNA using primers specific to each exon to test for intron incorporation into the mature pat-9 mRNA (Figure 2C). As predicted by the gene sequence, the point mutation resulted in mis-splicing of exon 3 to exon 4 resulting in the inclusion of the third intron (Figure 2C). DNA sequencing of this mis-spliced product confirmed the inclusion of the intron. Based on the pat-9 cDNA sequence, the predicted amino acid sequence is truncated due a premature stop codon in the mutant pat-9 mRNA (Figure 2D). Also, sequencing identified an additional 6 nucleotides between predicted exon 2 and exon 3 (Figure 2A) indicating pat-9 is spliced differently from the gene model displayed on WormBase (http://www.wormbase.org). Together, this evidence provides unambiguous confirmation that the identity of pat-9 is T27B1.2.

\section{PAT-9 is localized to nuclei through all developmental stages}

To characterize the PAT-9 protein, PAT-9 expression was analyzed in embryos, larvae and adults using a functional pat-9::gfp transgene and confirmed by immunostaining for endogenous PAT-9 (Figure 3). Since the DNA used for rescuing the Pat-9 phenotype contained $3.3 \mathrm{~kb}$ of genomic sequence upstream of the translational start site, this region of DNA was considered to be sufficient to function as the pat-9 promoter and was therefore used to generate a pat-9 transgene expression plasmid. The transgene was designed to express full length PAT-9, with GFP fused to its amino terminus in the vector pPD118.20. Expression of PAT-9::GFP from this plasmid was first detected in the early embryo; by the 2-fold stage of development PAT-9::GFP localized exclusively to the 

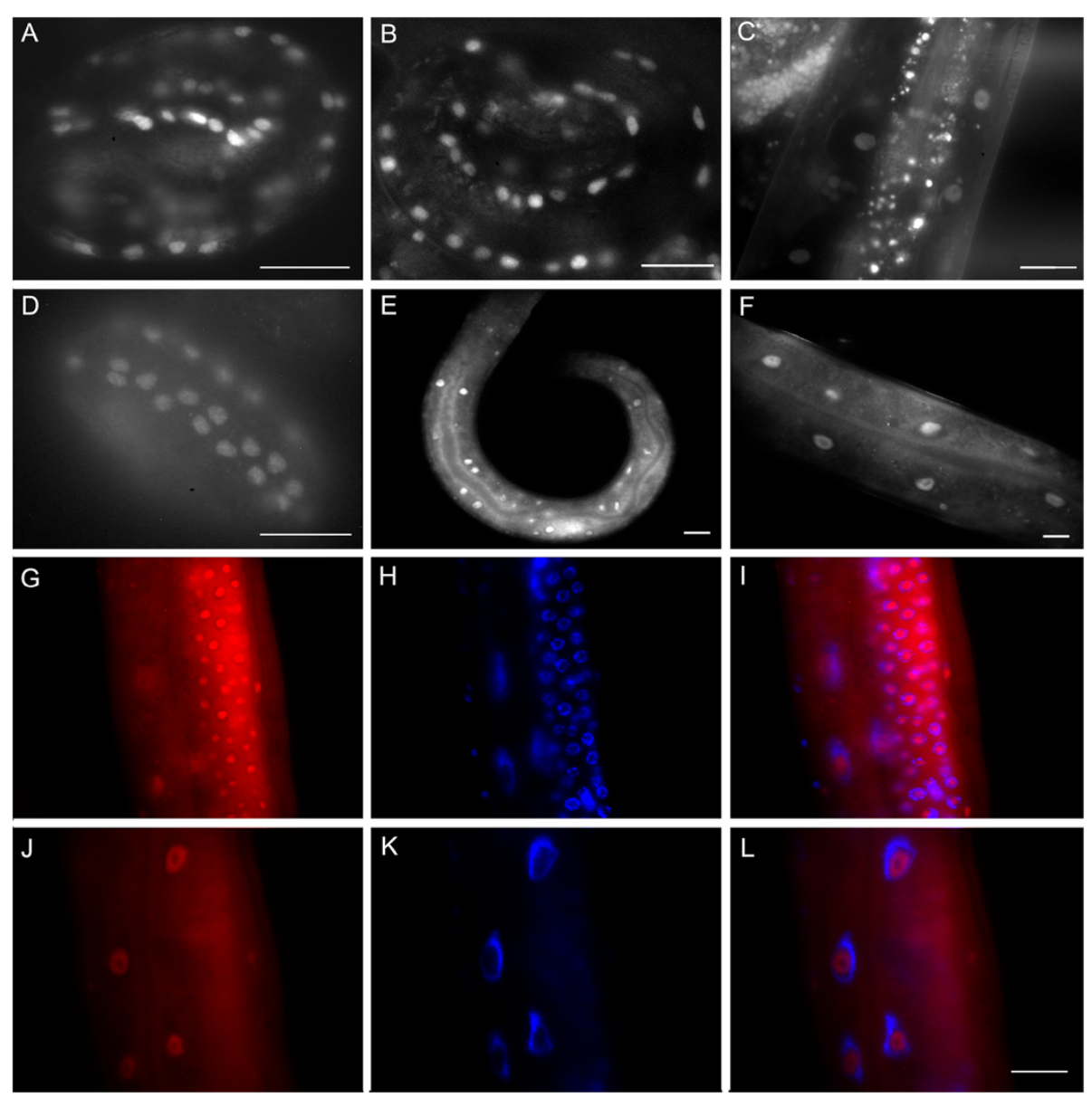

Figure 3 Characterization of PAT-9 expression. Localization of the PAT-9 protein as determined by use of a PAT-9::GFP translational fusion in the transgenic rescued pat-9(st558) stain. Fluorescence microscopy shows the localization of PAT-9::GFP within muscle nuclei in a A) 2-fold embryo, B) larva and C) adult animal. The anti-PAT-9 antibody stains body wall muscle nuclei of wild-type embryos (D), as well as the nuclei of young larvae $(\mathbf{E})$ and adults $(\mathbf{F})$; PAT-9 immunostaining shows that PAT-9 is expressed in germ-like nuclei in cytoplasmic syncytium of the $C$. elegans gonad (G). Endogenous PAT-9 (G and $\mathbf{J})$ is nuclear and localized to the DAPI-poor region as shown by (H and $\mathbf{K})$ DAPI staining, and (I and $\mathbf{L}$ ) merging with PAT-9 immunostaining. Bars $=10 \mu \mathrm{m}$.

body wall muscle nuclei in each body wall muscle quadrant (Figure 3A). PAT-9::GFP continued to be expressed in the cell nuclei of larvae and adults, and it became no longer exclusive to body wall muscle (Figure 3B and C). To confirm the transgenic expression pattern, antiserum against the PAT-9 carboxyl terminus, which does not contain the predicted zinc fingers, was generated (Additional file 1: Figure S1). The PAT-9 immunostaining of $\mathrm{N} 2$ animals showed a similar expression pattern to that seen with the pat-9 transgene. Endogenous PAT-9 was expressed from very early stages and throughout all developmental stages (Figure 3D, E and F), and in adults endogenous PAT-9 was predominantly nuclear and appeared excluded from the DAPI-poor nucleoli (Figure 3G-L). In addition to expression in body wall muscle, PAT-9 was observed in the germ cell-like nuclei of the cytoplasmic syncytium of the C. elegans gonad, where most nuclei are arrested in meiosis (Figure 3G, H and I).
Overall, the expression profile for PAT-9 is consistent with its involvement in muscle development; however, its apparent subcellular localization indicates that it is not a stable structural component of the sarcomere.

\section{PAT-9 has a nuclear localization signal (NLS)}

Analysis of the PAT-9 sequence for putative conserved domains using the Pfam protein conserved domain database (http://www.pfam.org) identified 3 putative C2H2 zinc fingers (aa 86-106, aa 112-136, aa 142-164), but no predicted NLS despite the strong nuclear localization of PAT-9. However, further analysis identified one region near the middle of the protein (aa 223-232) that contained five lysines (K) and an arginine (R) (Figure 4A) spaced so as to adhere to two proposed optimal consensus sequence requirements $[K(R / K) X(R / K)]$ and $[\mathrm{KR}(\mathrm{R} / \mathrm{X}) \mathrm{K}]$ of a classical NLS [21] suggesting this could be the PAT-9 NLS. To experimentally identify the PAT-9 

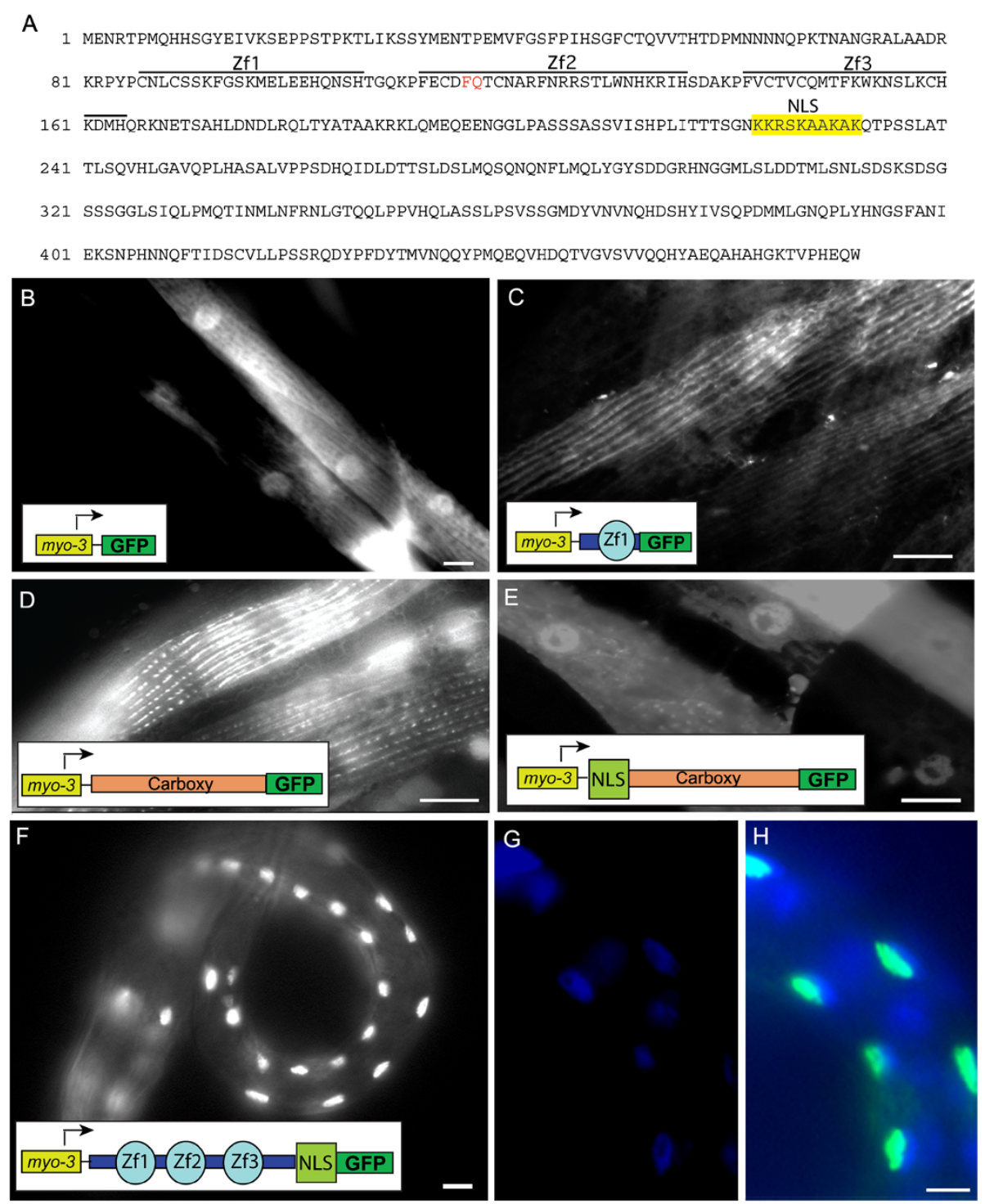

Figure 4 PAT-9 contains a lysine/arginine-rich NLS. A) The amino acid sequence predicted from the pat-9 cDNA cloned from N2 worms is shown. Each of the three putative zinc fingers is underlined. The additional two amino acids not present in the WormBase model are shown in red. The deduced NLS is highlighted in yellow. B-H) Plasmids expressing truncations of PAT-9 fused to GFP under control of the myo-3 promoter

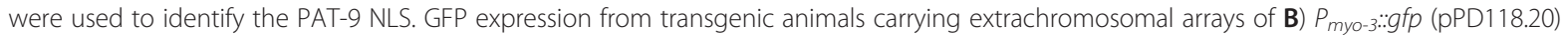
expressing GFP alone, C) $P_{\text {myo-3:: }}$ pat-9zf1::gfp, containing the amino terminal sequence and the first zinc finger of PAT-9, D) $P_{m y o-3:: p a t-9}$ C::.gfp containing only the carboxyl terminal sequence, E) $P_{\text {myo-3:: }}$ at-9CNLS::gfp containing the carboxyl terminal sequence with the putative NLS, F)

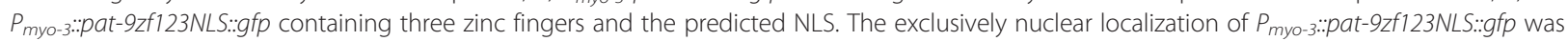
confirmed by G) DAPI staining and H) merging of GFP and DAPI images. Bars $=10 \mu \mathrm{m}$.

NLS, a series of truncations of pat-9 were generated and fused to GFP for transgenic expression analysis. The myo-3 promoter was used to specifically express all transgenes in body wall muscle. A transgenic line with $P_{m y o-3:: g f p}$ (pPD118.20) was used as a control; images of this show GFP localization throughout the muscle cell, including the nucleus, which is as expected since GFP enters the nucleus in the absence of an NLS due to its small size (Figure 4B). Transgenic animals expressing $P_{\text {myo-3 }:: z f 1:: g f p \text {-containing the amino terminal sequence }}$ and the first putative zinc finger, or $P_{m y o-3:: p a t-9 ~ C:: g f p-}$ containing the carboxyl terminal half of PAT-9, localized exclusively to the cytoplasm suggesting that there was no NLS in these regions (Figure $4 \mathrm{C}$ and D). Interestingly, both of these truncated PAT-9::GFP fusions showed an apparent sarcomeric localization, which may suggest a transient sarcomeric localization for PAT-9 prior to shuttling into the nucleus similar to UNC-95, UNC-97, UNC-98, and ZXY-1. However, when the putative NLS was added to the carboxyl terminal half of PAT-9, the 
GFP fusion protein strongly localized to the nucleus (Figure 4, compare D with E). Since the GFP fusion proteins are too large to passively diffuse into the nucleus, the observed nuclear localization must have been achieved through active import. Furthermore, GFP fused to an amino terminal portion of PAT-9 that includes the same putative NLS localized exclusively to muscle nuclei (Figure 4F-H). Taken together, the ten amino acid stretch containing the hypothetical NLS does indeed function as the PAT-9 NLS.

\section{PAT-9 associates with gene promoters}

The nuclear localization of PAT-9 along with the identification of 3 putative $\mathrm{C} 2 \mathrm{H} 2$ zinc fingers in pat-9 suggests that PAT-9 could be a nuclear $\mathrm{C} 2 \mathrm{H} 2$ zinc finger transcription factor that localizes to promoters and regulates gene expression. A yeast one-hybrid screen aimed at identifying $C$. elegans regulatory sequences bound by transcription factors predicted five promoters that could be bound by PAT-9/ZTF-19 [22]. To verify these potential interactions in vivo, chromatin immunoprecipitation (ChIP) experiments were performed using the PAT-9 polyclonal antibody. Two of the candidate promoters, $d a f-3$ and $t b x-2$, were chosen for ChIP experiments as well as a control gene, frg-1, not known to bind PAT-9. Cultures of mixed stage N2 animals were collected, fixed with formaldehyde, and used for ChIP with either PAT-9 antibody or normal rabbit serum (NRS). The associated DNAs were extracted, purified, and subjected to quantitative real-time PCR (qPCR). The cross threshold $(\mathrm{Ct}$ ) values (of which a smaller $\mathrm{Ct}$ number indicates a larger amount of the target DNA in the original sample) were determined for each promoter and the control, and the fold enrichment for each DNA in the PAT-9 ChIPs compared with the DNA in NRS ChIPs were calculated (Figure 5). Both the daf-3 (37.8 fold) and $t b x-2$ (18.1 fold) DNAs were strongly enriched in the PAT-9 ChIPs indicating that PAT-9 was highly bound to their promoters in vivo. Surprisingly, frg- 1 was slightly enriched (5.5 +/ 3.7 fold) in the PAT-9 ChIPs, despite not being a one-hybrid hit. Potentially accounting for this, frg-1, similar to pat-9, is expressed throughout development and within muscle, and may, in fact, be regulated by PAT-9 [6]. Overall, our results indicate that PAT-9 interacts with gene promoters in vivo and likely regulates the expression of additional genes not identified by the one-hybrid approach, at least one of which must account for the Pat-9 phenotype.

\section{PAT-9 is required for the assembly of functional muscle attachments}

In Pat mutants, including pat-9, actin and myosin filaments are not recruited properly to muscle attachment sites suggesting that the assembly of muscle attachments

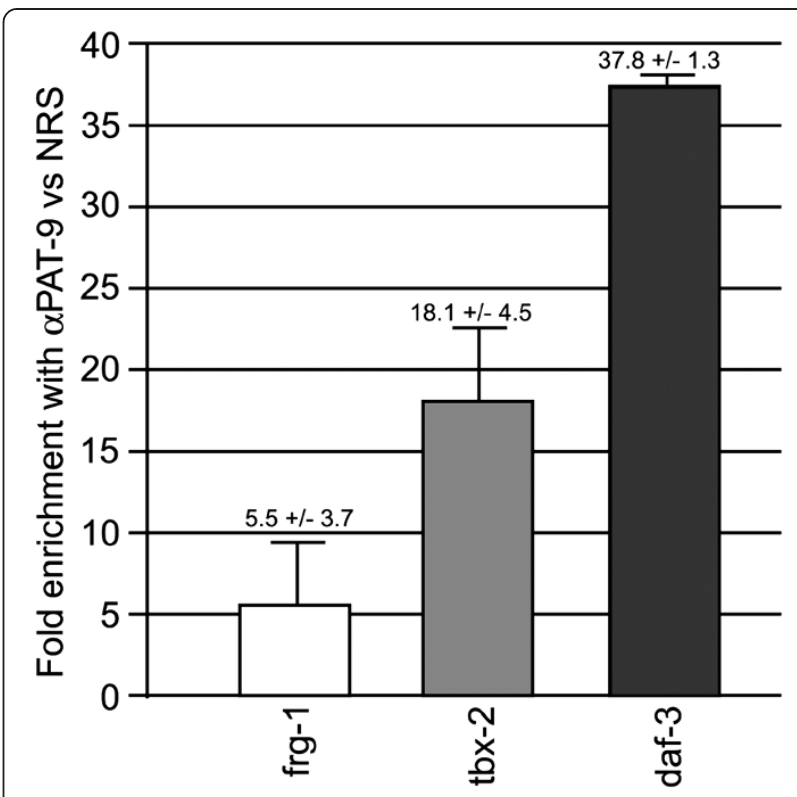

Figure 5 PAT-9 is associated with gene promoters in vivo. ChIP experiments show enrichment of the indicated 5' promoter DNA with the aPAT-9 antibody compared with NRS control as assayed by qPCR. The tbx-2 and daf-3 bars represent the results from three independent ChIP experiments and the frg- 1 bar represents the results from six independent ChIP experiments. All qPCRs were performed as five replicates to generate one data point. Fold enrichment was calculated as 2e (Ct NRS - Ct PAT-9)

is disrupted. The PAT-9 nuclear localization and ChIP data suggests a gene regulatory role for PAT-9 in the expression of genes encoding muscle attachment structural components. Thus, the expression and localization of known muscle attachment proteins was investigated in pat-9 mutants. The pat-9(st558) allele was used to ablate functional PAT-9 expression and the embryos were stained with antibodies to components of muscle attachments (Figure 6). Immunostaining showed that UNC-52/perlecan (Figure 6A'), PAT-3/integrin (Figure 6B'), PAT-4/ILK (Figure 6C'), DEB-1/vinculin (Figure 6D'), and myosin (Figure 6E'), were all expressed in pat-9(st558) embryos. In wild type embryos, PAT-3/integrin, PAT-4/ILK, and DEB$1 /$ vinculin are located in ordered arrays of dense bodies and $\mathrm{M}$ lines (Figure 6A-E). In contrast, in pat-9(st558) mutant embryos, these proteins are not well organized and UNC-52/perlecan was not located in a continuous strip in the basal lamina of pat-9(st558) mutants. Muscle attachments have a distinct assembly pathway beginning with proteins such as UNC-52/perlecan [2]; therefore, when early components fail to properly assemble, then downstream components such as PAT-3/integrin, PAT-4/ ILK, DEB-1/vinculin and myosin are not well organized either. In wild-type animals, myofilaments are organized, recognizable, and attached to the muscle cell membrane through muscle attachments forming two arrays of muscle cells that are parallel in each body wall muscle quadrant. 

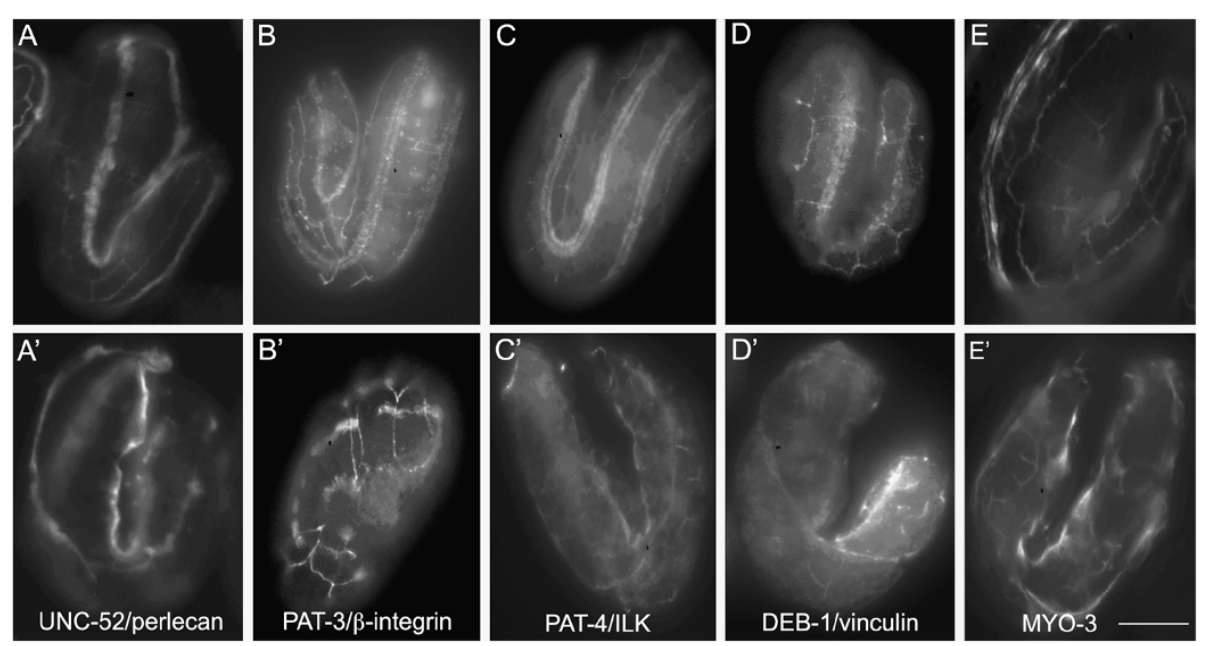

Figure 6 Muscle attachment sites are disrupted in pat-9 mutants. N2 (A, B, C, D and E) and pat-9(st558) (A', B', $C^{\prime} D^{\prime}$ and $\left.E^{\prime}\right)$ embryos were stained with monoclonal antibodies to UNC-52/perlecan (A and $\left.\mathbf{A}^{\prime}\right)$, PAT-3/beta-integrin (B and $\left.\mathbf{B}^{\prime}\right)$, PAT-4/ILK (C and $\left.\mathbf{C}^{\prime}\right)$, DEB-1/vinculin (D and $\left.\mathbf{D}^{\prime}\right)$ and MYO-3/MHC-A (E and $\mathbf{E}^{\prime}$ ). All five proteins are expressed in pat-9(st558) embryos indicating PAT-9 does not regulate these genes. Arrows show the structures stained by each antibody. All the animals were co-stained with monoclonal antibody $\mathrm{MH} 27$ to visualize the hypodermal cell junctions, enabling the identification of the orientation and age of the embryos. Bar $=10 \mu \mathrm{m} .3^{\text {rd }}$ exon.

In many pat-9 mutant embryos, the dorsal body-wall muscle quadrants have pulled away from the body wall: direct evidence that the muscle attachments have not been properly formed. Furthermore, myosin filaments are not recruited properly in pat-9(st558) mutant embryos. Thus, although many components of muscle attachments are expressed in these mutants, they often fail to form functional muscle attachments and seem to require PAT-9 for their proper assembly.

\section{Discussion}

We have shown that the pat-9 gene is the predicted gene T27B1.2, renamed $z t f-19$, which encodes a nuclear $\mathrm{C} 2 \mathrm{H} 2$ type zinc finger transcription factor. Thus, PAT9 is the only exclusively nuclear protein among all pat genes that have been identified and characterized. In mammals, many DNA-binding transcriptional regulators contain $\mathrm{C} 2 \mathrm{H} 2$ zinc fingers [23]. Similarly, in $C$. elegans $\mathrm{C} 2 \mathrm{H} 2$ zinc finger transcription regulators play critical roles in many biological aspects, including body wall muscle development, synaptic transmission, and egg-laying behavior and fertility [15,24,25]. The characterization of PAT-9 suggests it functions as a transcription factor for one or more factors required in muscle attachment site assembly. Lending support to this hypothesis, a yeast one-hybrid binding assay showed that PAT-9/ZTF-19 bound to five promoters, daf-3, tbx-2, $\operatorname{cog}-1$, let-7, and mir-76 [22]. Furthermore, two of the genes, $d a f-3$ and $t b x-2$, were shown to be bound by PAT-9 in vivo. Interestingly, both genes are transcriptional regulators themselves involved in C. elegans pharynx development, while daf-3 is additionally involved in dauer formation, indicating that PAT-9 has multiple roles during development including roles outside of body wall muscle development [26-28].

TBX-2 is a transcription factor specifically required for development of ABa-derived pharyngeal muscles [26]. TBX-2 is expressed from early embryo to adult stages and $t b x-2:: g f p$ expression was also observed in body wall muscles at the comma stage and later, in 2- to 3-fold embryos similar to the expression of pat-9. As with pat-9, there is a muscle defect in $t b x-2$ mutants at the embryo stage. Interestingly, RNAi analysis reveals that $t b x-2$ is critical for early larval development and normal locomotion. These animals have a less severe phenotype than the pat-9 RNAi animals that have embryo lethality and larval arrest, but are still consistent with TBX-2 being a downstream target of PAT-9 [29,30].

DAF-3 is a transcriptional regulator negatively regulated by TGF- $\beta$ signaling [28]. Loss of DAF-3 by genetic null does not result in locomotion or embryonic lethality phenotypes, but rather, dauer defective animals [31]. The other predicted targets of PAT-9/ZTF-19 are unlikely candidates for producing the Pat phenotype; $\operatorname{cog}-1$ is expressed in neurons [32], let-7 is a heterochronic switch gene only expressed in L3, L4 and adults [33], and the mir-76 microRNA is expressed in early embryos and adult neurons but not body wall muscle lineages [34]. This expression information along with the ChIP data has enabled us to hypothesize that PAT-9 is involved in the regulation of both $t b x-2$ and $d a f-3$ expression, however, neither gene is responsible for the Pat phenotype of pat-9 mutants, leaving the critical downstream target(s) of PAT-9 yet to be identified. 
PAT-9 is required for proper organization of myofilaments and for recruitment to the $M$ line. In pat-9 mutants, UNC-52/perlecan, the most upstream molecule of the muscle attachment assembly pathway, is present in the basal lamina; however, PAT-3/integrin is not well organized at the basal membrane. Subsequently, PAT-4/ ILK and DEB-1/vinculin are not recruited properly to the nascent attachment sites. The correct assembly of dense bodies and $M$ lines are dependant upon the recruitment of each protein component in a distinct order, such that failure of one protein to assemble hinders the recruitment of all other proteins downstream in the pathway $[16,17]$. Therefore it is reasonable to make the prediction that the molecular components at muscle attachment sites downstream of integrin, including UNC112, UNC-97/ PINCH, and PAT-6/actopaxin, would not be assembled properly at the attachment sites of pat-9 mutants. In both pat-4 and pat-6 mutants, the PAT-3/integrin is not affected, since both proteins are downstream of PAT-3 in the assembly pathway. However, in pat-9 mutants integrin is not properly organized at the basal membrane, which directly causes the disruption of the integrity of the attachment complex and expression of UNC-52 is not enough to initiate the assembly of muscle at attachment sites. Since integrins have two-way signaling, the lack of PAT-9 causes the loss of a critical inside out signaling mechanism, compounding the Pat-9 phenotype.

\section{Conclusions}

The assembly of proteins forming muscle attachments is a highly ordered process, but is also regulated at the transcriptional level by transcription factors. Our study shows that PAT-9/ZTF-19 is indispensable for establishing and maintaining the integrity of muscle attachment sites and most likely functions at the level of transcription regulation.

\section{Methods}

\section{Strains and genetics}

Standard methods were used for culturing C. elegans [35]. The following strains were used: wild-type worms were N2 strain of the Bristol variety; RW1385 ( $m n D p 1$ $(X ; V) /+V ;$ unc-3(e151) pat-9(st558) X). SNP mapping was performed as described [36]. RW1385 worms were mated with Hawaiian CB4856 males and Unc non-Pat recombinants were isolated and used for PCR and RFLP analysis around known SNPs.

Because homozygote pat-9 is lethal, a segment of duplication of the $\mathrm{X}$ chromosome $(m n D p 1)$ containing wildtype pat-9 and unc-3 genes was fused to one of its $\mathrm{V}$ chromosomes. This strain segregates $25 \%$ pats, $25 \%$ early arrested embryos containing two duplications and 50\% normal progeny with the same genotype as RW1385. For the pat-9 rescue line $(+/+V$; unc-3(e151) pat-9(st558) X; pat-9::gfp), the rescue animal lost the duplication $m n D p 1$ and is homozygous for unc-3 and pat-9. The Pat phenotype was rescued by a wild-type pat- 9 gene in an extrachromosomal array and was homozygous for unc-3, yielding an Unc phenotype and coiled tail. The extrachromosomal arrays contain the dominant transformation marker pRF4(rol-6); therefore the rescued strain segregates either rollers with coiled tail or Pats.

\section{Molecular biology}

All PCR fragments used to generate expression plasmids were first subcloned into pGEM-T easy (Promega) and sequenced. All oligonucleotide primers are listed in Additional file 1: Table S3. Cosmid T27b1 was verified to contain the whole T27b1.2 gene by sequencing with primers BW-580, BW-581, BW-582 and BW-583. For the initial transformation rescue experiment, the pat-9 $3.3 \mathrm{~kb}$ promoter and coding region were amplified separately with primer pairs (BW-608, BW-610), and (BW-611, BW-612) respectively, using cosmid T27b1 as the template. Two PCR fragments have a $500 \mathrm{bp}$ overlap. The initial pat-9 full-length cDNA was generated by RT-PCR using Superscript III/Platinum Taq one-step RT-PCR system (Invitrogen) and primer pair (BW-631, BW-632). Total RNA was isolated from N2 worms using a TRIZOL (Invitrogen) based method with slight modification: 800 ul Trizol was added to 200ul packed worms, vortexed, and incubated at room temperature for $20 \mathrm{~min}$. The reaction was centrifuged at full speed at $4^{\circ} \mathrm{C}$ for $10 \mathrm{~min}$, the supernatant containing the RNA was removed to a new tube, purified as per manufacturers instructions, and RNA was suspended in $10 \mu \mathrm{l}$ DEPC- $\mathrm{H}_{2} \mathrm{O}$.

To study the expression and localization of PAT-9, a pat-9::gfp fusion was constructed as follows. First, the $3.3 \mathrm{~kb}$ pat-9 promoter was PCR-amplified using primers BW-613 and BW-630. This fragment was digested with PstI and KpnI, and then replaced the myo-3 promoter of pPD118.20 (Addgene, Fire Lab C. elegans Vector Kit), to make $P_{\text {pat- } 9:: g f p \text {. The pat-9 genomic fragment including }}$ $980 \mathrm{bp}$ downstream of the $6^{\text {th }}$ exon was PCR-amplified using primers BW631 and BW596, digested with KpnI and DraIII, and then replaced the KpnI - DraIII region of

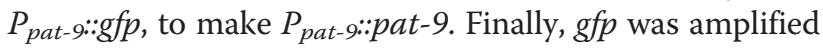
and digested with KpnI, and inserted into $P_{\text {pat-9:::pat-9, to }}$

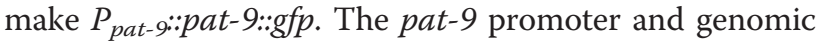
coding region were amplified using cosmid T27b1 as a template; $g f p$ was amplified using vector pPD118.20 as a template.

The following transgene plasmid constructs for NLS identification were made in the pPD118.20 vector with gfp fused in-frame to the carboxyl terminus of the pat-9 coding sequence. Deletions of pat-9 were amplified from the pat- 9 cDNA and cloned in-frame with $g f p$ by NotI 
and KpnI digestion. The following primer pairs were used for PCR amplification: pat-9 C (primer 2 and primer 3), pat-9CNLS (primer), pat-9zf1(PJ-101, PJ-105), pat-9zf123NLS (primer 1 and primer 4). The above PCR fragments were digested with NotI and KpnI and inserted into the vector pPD118.20 NotI and KpnI site to make

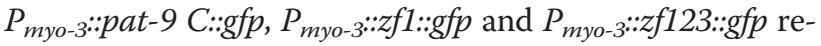
spectively. All plasmids were verified by sequencing. Transgenic lines were made using the standard microinjection approach with pRF4(rol-6) as an injection marker.

\section{PAT-9 antibody}

A partial pat-9 cDNA fragment (carboxyl terminal, amino acids 176-470) was PCR amplified (using primers PJ-117 and PJ-118) from the pat-9 cDNA, digested with NdeI and NotI, sub-cloned into a NdeI and NotI digested pET23a plasmid (Novagen), and transformed into E. coli BL21(DE3) bacteria for expression. Recombinant protein was purified under denaturing conditions using Talon Resin (Clontech) as per manufacturer's instructions, dialyzed with PBS, and used as an immunogen. Rabbit polyclonal antibodies were generated at the Immune Resource Center at the University of Illinois at Urbana-Champaign. Antiserum was affinity purified against recombinant PAT-9 C-terminal protein covalently linked to cyanogen bromide-activated sepharose (GE Healthcare) and analyzed by immunoblotting for specificity (Additional file 1: Figure S2).

\section{RNA interference}

RNAi for all of the nine candidate pat-9 genes were performed as previously described by dsRNA injection $[17,37]$ and additionally for T27b1.2 by feeding bacteria induced to express double-stranded RNA to the worms [38]. The cDNA clones $y k 64 f 5, y k 414 e 5, y k 443 f 12, y k 483 d 11$, $y k 668 g 10, y k 782 b 11$, and $y k 839 g 10$ were kindly provided by Dr. Yuji Kohara, National Institute of Genetics, Mishima, Shizuoka, Japan. RT-PCR from N2 RNA was performed to obtain the cDNAs for T25D1.2 (BW-570, BW-571) and F59C12.3 (BW-572, BW-573). For injection RNAi, sense and antisense RNA were generated by in vitro transcription using T3 and T7 RNA polymerase (Promega). The RNAs were mixed $(1 \mu \mathrm{g} / \mu \mathrm{l})$, injected into N2 hermaphrodites, and eggs laid between 12 - $48 \mathrm{~h}$ after injection were scored for the Pat phenotype. For feeding RNAi, the T27b1.2 cDNA was cloned between the two T7 promoters of vector pL4440 (Open Biosystems). The plasmid was transformed into competent HT115(DE3) bacteria, grown up from single colonies in 2x YT media containing tetracycline and kanamycin, and expression of dsRNA was induced with $1 \mathrm{mM}$ IPTG when the culture reached OD600 $=0.3 \sim 0.4$. NGM plates, made fresh with $50 \mu \mathrm{g} / \mathrm{ml}$ kanamycin, $12.5 \mu \mathrm{g} / \mu \mathrm{l}$ tetracycline and $0.4 \mathrm{mM}$ IPTG, were seeded with the induced cultures and set at room temperature overnight. L4-stage hermaphrodite worms were placed on the plates and incubated for $48 \mathrm{~h}$ at $18^{\circ} \mathrm{C}$ after which 5 worms were transferred separately onto plates seeded with the same bacteria and allowed to lay eggs for $24 \mathrm{~h}$ at $18^{\circ} \mathrm{C}$ before being removed. Progeny from both the first plates and second plates were scored for a Pat phenotype and any other developmental abnormalities after another $24 \mathrm{~h}$.

\section{ChIP}

The ChIP experiments were performed essentially as described [39]. N2 worms were grown in $500 \mathrm{ml} \mathrm{S}$ medium at $20^{\circ} \mathrm{C}$ with shaking-producing mixed stage cultures, collected, washed, and fixed with formaldehyde. Approximately $0.4 \mathrm{ml}$ of packed N2 worms were used for each ChIP experiment and each ChIP replicate experiment started with a new liquid culture. After fixation, soluble chromatin was generated by sonication, resulting in an average of 750-500 bp DNA fragments as determined by agarose gel. For the immunoprecipitation step, $10 \%$ of the soluble chromatin was removed and used as input control and the remaining chromatin was divided into two equal pools. The PAT-9 was added (1:100) to one tube and NRS (1:100) was added to the second sample as a control for non-specific binding. IPs proceeded overnight at $4^{\circ} \mathrm{C}$ with end-over-end rotation. The immune/chromatin complexes were collected using prelocked protein A/G agarose (Santa Cruz Biotech). After washing extensively, the bound DNA was purified and subjected to qPCR using SYBR Green and a BioRad I-Cycler. PCR primers were designed to the $5^{\prime}$ regulatory regions of frg-1 (PJ-111, PJ-112), daf-3 (PJ113, PJ-114), and $t b x-2$ (PJ-115, PJ-116), and tested for conditions resulting in a single PCR product of the expected size.

\section{Embryo immunostaining}

Populations of embryos were fixed and stained as previously described [40]. The following monoclonal antibodies were obtained from the Developmental Studies Hybridoma Bank and diluted as indicated: MH2 (1:100), MH25 (1:250), MH24 (1:200), and MH27 (1:1500) (developed by R. H. Waterston), and 5-6 (1:200) (developed by H. F. Epstein). The mouse polyclonal PAT-4 antibody [16] and rabbit polyclonal PAT-9 antibody were generated in the lab as described. Antibodies were diluted for staining in PBS supplemented with $0.5 \%$ tween-20 and 30\% normal goat serum. Affinity-purified goat anti-mouse IgG conjugated to rhodamine, diluted 1:100 (Chemicon International) or AlexaFluor594 goat anti-rabbit IgG, diluted 1:800 (Invitrogen Corp) was used as a secondary antibody. 


\section{Adult worm immunostaining}

Immunostaining on adult animals was essentially as described [41]. Mixed stage N2 worms were harvested and washed thoroughly in PBS to remove bacteria. Worms were suspended in $4 \%$ paraformaldehyde in $100 \mathrm{mM}$ sodium phosphate buffer and quick frozen on dry ice. After thawing on ice, animals were incubated on ice for $1 \mathrm{~h}$, washed three times in $1 \%$ triton $\mathrm{X}-100,100 \mathrm{mM}$ tris $(\mathrm{pH} 7.5)$, then incubated in $1 \%$ triton $\mathrm{X}-100,100 \mathrm{mM}$ tris $(\mathrm{pH} 7.5)$ and $1 \% \beta$-mercaptoethanol at $37^{\circ} \mathrm{C}$ for $2 \mathrm{~h}$. Worms were washed three times in $10 \mathrm{mM} \mathrm{NaBO}_{3}$ (pH 9.2), incubated for $1 \mathrm{~h}$ in $10 \mathrm{mM} \mathrm{NaBO}_{3}+0.3 \% \mathrm{H}_{2} \mathrm{O}_{2}$, washed 3 times with $10 \mathrm{mM} \mathrm{NaBO}_{3}$ (pH 9.2) and stored for further processing in AbA buffer $(1 \times \mathrm{PBS}, 0.1 \%$ triton $\mathrm{X}-100,1 \% \mathrm{BSA}, 0.05 \% \mathrm{NaN}_{3}$ ). The PAT-9 antibody was diluted 1:500 in AbA buffer, incubated overnight at $4^{\circ} \mathrm{C}$, washed three times with $\mathrm{AbB}$ buffer $(1 \times \mathrm{PBS}, 0.1 \%$ triton $\mathrm{X}-100,0.1 \% \mathrm{BSA}, 0.05 \% \mathrm{NaN}_{3}$ ) and incubated with secondary antibody (Alexa 488 goat anti-rabbit) at $4^{\circ} \mathrm{C}$ overnight. After washing with $\mathrm{AbB}$ three times, the pellets were mounted on slides for fluorescence microscopy.

\section{Additional file}

Additional file 1: Characterization of the affinity purified PAT-9 rabbit polyclonal antibody. Western blots of total protein extract from A) pat-9 or N2 embryos, or B) pat9::gfp transgenic embryos probed with the PAT-9 polyclonal antibody or GFP antibody as indicated. Arrowhead indicates PAT-9 and arrow indicates PAT-9::GFP fusion protein. Table S1. SNP Mapping results. Table $\mathbf{S} 2$. Nine candidate pat-9 genes based on SAGE data. Table S3. Oligonucleotide primers.

\section{Competing interests}

The authors declare that they have no competing interests.

\section{Acknowledgements}

The authors thank Dr. Yuji Kohara (the National Institute of Genetics, Mishima, Shizuoka, Japan) for providing cDNA clones, and Dr. Andy Fire (Stanford University) and Dr. Phil Newmark (University of Illinois at Urbana-Champaign) for plasmid vectors. The monoclonal antibodies were developed by R. H. Waterston and obtained from the Developmental Studies Hybridoma Bank developed under the auspices of the NICHD and maintained by The University of lowa, Department of Biology, lowa City, IA 52242. This work was funded by a National Institutes of Health Grant [RO1HD38464] to BDW and institutional support from the University of Illinois at Urbana-Champaign for BDW and PL.

\section{Authors' contributions}

BDW designed the overall study and performed the original Pat screen that isolated pat-9. All authors performed experiments. QL, TIJ, RAB and PLJ drafted the manuscript. All authors read and approved the final manuscript.

Received: 17 April 2012 Accepted: 22 May 2012

Published: 22 May 2012

\section{References}

1. Moerman DG, Williams BD: Sarcomere assembly in C. elegans muscle. WormBook 2006, :1-16.

2. Qadota H, Benian GM: Molecular structure of sarcomere-to-membrane attachment at M-Lines in C. elegans muscle. J Biomed Biotechnol 2010, 2010:864749.

3. Mackenzie JM Jr, Garcea RL, Zengel JM, Epstein HF: Muscle development in Caenorhabditis elegans: mutants exhibiting retarded sarcomere construction. Cell 1978, 15:751-762.
4. Francis GR, Waterston $\mathrm{RH}$ : Muscle organization in Caenorhabditis elegans: localization of proteins implicated in thin filament attachment and I-band organization. J Cell Biol 1985, 101:1532-1549.

5. Hresko MC, Williams BD, Waterston $\mathrm{RH}$ : Assembly of body wall muscle and muscle cell attachment structures in Caenorhabditis elegans. J Cell Biol 1994, 124:491-506.

6. Liu Q, Jones TI, Tang WW, Brieher WM, Jones PL: Facioscapulohumeral muscular dystrophy region gene-1 (FRG-1) is an actin bundling protein associated with muscle attachment sites. J Cell Sci 2010, 123:1116-1123.

7. Lecroisey C, Segalat L, Gieseler K: The C. elegans dense body: anchoring and signaling structure of the muscle. J Muscle Res Cell Motil 2007, 28:79-87.

8. Hanel ML, Sun CY, Jones TI, Long SW, Zanotti S, Milner D, Jones PL: Facioscapulohumeral muscular dystrophy (FSHD) region gene 1 (FRG1) is a dynamic nuclear and sarcomeric protein. Differentiation 2010, 2:107-118.

9. Barstead RJ, Waterston RH: The basal component of the nematode densebody is vinculin. J Biol Chem 1989, 264:10177-10185

10. Williams BD, Waterston RH: Genes critical for muscle development and function in Caenorhabditis elegans identified through lethal mutations. J Cell Biol 1994, 124:475-490.

11. Waterston RH, Thomson JN, Brenner S: Mutants with altered muscle structure of Caenorhabditis elegans. Dev Biol 1980, 77:271-302.

12. Lecroisey C, Martin E, Mariol MC, Granger L, Schwab Y, Labouesse M, Segalat $L$, Gieseler K: DYC-1, a protein functionally linked to dystrophin in Caenorhabditis elegans is associated with the dense body, where it interacts with the muscle LIM domain protein ZYX-1. Mol Biol Cell 2008, 19:785-796.

13. Broday L, Kolotuev I, Didier C, Bhoumik A, Podbilewicz B, Ronai Z: The LIM domain protein UNC-95 is required for the assembly of muscle attachment structures and is regulated by the RING finger protein RNF-5 in C. elegans. J Cell Biol 2004, 165:857-867.

14. Hobert O, Moerman DG, Clark KA, Beckerle MC, Ruvkun G: A conserved LIM protein that affects muscular adherens junction integrity and mechanosensory function in Caenorhabditis elegans. J Cell Biol 1999, 144:45-57.

15. Mercer KB, Flaherty DB, Miller RK, Qadota H, Tinley TL, Moerman DG, Benian GM: Caenorhabditis elegans UNC-98, a C2H2 Zn finger protein, is a novel partner of UNC-97/PINCH in muscle adhesion complexes. Mol Biol Cell 2003, 14:2492-2507.

16. Mackinnon AC, Qadota H, Norman KR, Moerman DG, Williams BD: C. elegans PAT-4/ILK functions as an adaptor protein within integrin adhesion complexes. Curr Biol 2002, 12:787-797.

17. Lin X, Qadota H, Moerman DG, Williams BD: C. elegans PAT-6/actopaxin plays a critical role in the assembly of integrin adhesion complexes in vivo. Curr Biol 2003, 13:922-932.

18. Norman KR, Cordes S, Qadota H, Rahmani P, Moerman DG: UNC-97/PINCH is involved in the assembly of integrin cell adhesion complexes in Caenorhabditis elegans body wall muscle. Dev Biol 2007, 309:45-55.

19. Williams BD, Schrank B, Huynh C, Shownkeen R, Waterston RH: A genetic mapping system in Caenorhabditis elegans based on polymorphic sequence-tagged sites. Genetics 1992, 131:609-624.

20. Meissner B, Warner A, Wong K, Dube N, Lorch A, McKay SJ, Khattra J, Rogalski T, Somasiri A, Chaudhry I, et al: An integrated strategy to study muscle development and myofilament structure in Caenorhabditis elegans. PLOS Genet 2009, 5:e1000537.

21. Marfori M, Mynott A, Ellis JJ, Mehdi AM, Saunders NF, Curmi PM, Forwood JK, Boden M, Kobe B: Molecular basis for specificity of nuclear import and prediction of nuclear localization. Biochim Biophys Acta 2010, 1813:1562-1577.

22. Barrasa MI, Vaglio P, Cavasino F, Jacotot $L$, Walhout AJ: EDGEdb: a transcription factor-DNA interaction database for the analysis of $C$. elegans differential gene expression. BMC Genomics 2007, 8:21.

23. Iuchi S: Three classes of $\mathrm{C} 2 \mathrm{H} 2$ zinc finger proteins. Cell Mol Life Sci 2001, 58:625-635.

24. Lakowski B, Eimer S, Gobel C, Bottcher A, Wagler B, Baumeister R: Two suppressors of sel-12 encode $\mathrm{C} 2 \mathrm{H} 2$ zinc-finger proteins that regulate presenilin transcription in Caenorhabditis elegans. Development 2003, 130:2117-2128.

25. Wang Y, Gracheva EO, Richmond J, Kawano T, Couto JM, Calarco JA, Vijayaratnam $\mathrm{V}$, Jin $\mathrm{Y}$, Zhen $\mathrm{M}$ : The $\mathrm{C} 2 \mathrm{H} 2$ zinc-finger protein SYD-9 is a putative posttranscriptional regulator for synaptic transmission. Proc Natl Acad Sci U S A 2006, 103:10450-10455. 
26. Roy Chowdhuri S, Crum T, Woollard A, Aslam S, Okkema PG: The T-box factor TBX-2 and the SUMO conjugating enzyme UBC-9 are required for ABa-derived pharyngeal muscle in C. elegans. Dev Biol 2006, 295:664-677.

27. Thatcher JD, Haun C, Okkema PG: The DAF-3 Smad binds DNA and represses gene expression in the Caenorhabditis elegans pharynx. Development 1999, 126:97-107.

28. Patterson Gl, Koweek A, Wong A, Liu Y, Ruvkun G: The DAF-3 Smad protein antagonizes TGF-beta-related receptor signaling in the Caenorhabditis elegans dauer pathway. Genes Dev 1997, 11:2679-2690.

29. Sonnichsen B, Koski LB, Walsh A, Marschall P, Neumann B, Brehm M, Alleaume AM, Artelt J, Bettencourt P, Cassin E, et al: Full-genome RNA profiling of early embryogenesis in Caenorhabditis elegans. Nature 2005, 434:462-469.

30. Simmer F, Moorman C, van der Linden AM, Kuijk E, van den Berghe PV, Kamath RS, Fraser AG, Ahringer J, Plasterk RH: Genome-wide RNAi of C. elegans using the hypersensitive rrf-3 strain reveals novel gene functions. PLOS Biol 2003, 1:E12.

31. Vowels JJ, Thomas JH: Genetic analysis of chemosensory control of dauer formation in Caenorhabditis elegans. Genetics 1992, 130:105-123.

32. Palmer RE, Inoue T, Sherwood DR, Jiang LI, Sternberg PW: Caenorhabditis elegans cog-1 locus encodes GTX/Nkx6.1 homeodomain proteins and regulates multiple aspects of reproductive system development. Dev Biol 2002, 252:202-213

33. Reinhart BJ, Slack FJ, Basson M, Pasquinelli AE, Bettinger JC, Rougvie AE, Horvitz HR, Ruvkun G: The 21-nucleotide let-7 RNA regulates developmental timing in Caenorhabditis elegans. Nature 2000 403:901-906.

34. Martinez NJ, Ow MC, Reece-Hoyes JS, Barrasa MI, Ambros VR, Walhout AJ: Genome-scale spatiotemporal analysis of Caenorhabditis elegans microRNA promoter activity. Genome Res 2008, 18:2005-2015.

35. Brenner S: The genetics of Caenorhabditis elegans. Genetics 1974, 77:71-94.

36. Wicks SR, Yeh RT, Gish WR, Waterston RH, Plasterk RH: Rapid gene mapping in Caenorhabditis elegans using a high density polymorphism map. Nat Genet 2001, 28:160-164.

37. Fire A, Xu S, Montgomery MK, Kostas SA, Driver SE, Mello CC: Potent and specific genetic interference by double-stranded RNA in Caenorhabditis elegans. Nature 1998, 391:806-811.

38. Timmons $L$, Court $D L$, Fire A: Ingestion of bacterially expressed dsRNAs can produce specific and potent genetic interference in Caenorhabditis elegans. Gene 2001, 263:103-112.

39. Mukhopadhyay A, Deplancke B, Walhout AJ, Tissenbaum HA: Chromatin immunoprecipitation (ChIP) coupled to detection by quantitative realtime PCR to study transcription factor binding to DNA in Caenorhabditis elegans. Nat Protoc 2008, 3:698-709.

40. Barstead RJ, Kleiman L, Waterston RH: Cloning, sequencing, and mapping of an alpha-actinin gene from the nematode Caenorhabditis elegans. Cell Motil Cytoskeleton 1991, 20:69-78.

41. Finney M, Ruvkun $\mathrm{G}$ : The unc-86 gene product couples cell lineage and cell identity in C. elegans. Cell 1990, 63:895-905.

doi:10.1186/2045-3701-2-18

Cite this article as: Liu et al:: C. elegans PAT-9 is a nuclear zinc finger protein critical for the assembly of muscle attachments. Cell \& Bioscience 2012 2:18.

\section{Submit your next manuscript to BioMed Central and take full advantage of:}

- Convenient online submission

- Thorough peer review

- No space constraints or color figure charges

- Immediate publication on acceptance

- Inclusion in PubMed, CAS, Scopus and Google Scholar

- Research which is freely available for redistribution 